instabilities occurring in the same region of the tail.

The chief bar to progress in studying substorms is the difficulty of unravelling the complex magnetic data to identify them and their starting time. It has been established that the auroral electrojet index, which is a measure of currents flowing in the auroral ionosphere, is unreliable for this purpose. Most workers concede this difficulty, the exception being $\mathrm{C}$. McIlwain of the University of California, San Diego, who claims to be able to establish the beginning of a substorm to an accuracy of plus or minus five minutes and to have done so for over 3,000 substorms. The basis of Mcllwain's method is that the magnetospheric substorm corresponds to a sudden intensification of the electric fields in the magnetosphere which in turn produce an increase in the particle populations in the tail sector. These particle enhancements are reported by McIlwain to be clearly seen in the data acquired from the geosynchronous satellite ATS 5. Clearly important work will follow when the timings and details of these substorms are released.

Although much of the conference was devoted to the Earth's magnetosphere, Drs F. M. Neubauer (Technical University, Branschweig), N. Brice (Cornell University) and J. Warwick (University of Colorado) reported work on Jupiter's magnetosphere. Jupiter has a magnetic field which is about 10 gauss at the surface and gives rise to a much larger magnetosphere and associated radiation belts than those of the Earth. Estimates of the position of the forward boundary of the Jovian magnetosphere put it at a distance of about 50 Jupiter radii, compared with a distance of 10 Earth radii for the forward boundary of our magnetosphere.

\section{NUCLEAR STRUCTURE}

\section{Single-particle States}

from a Correspondent

AcCording to the independent particle shell model each nucleon in the nucleus moves in its own orbit, characterized by definite quantum numbers, and its spatial distribution is given by its wavefunction. The orbits are filled starting from those of lowest energy and subject to the Pauli principle that no two particles can have the same quantum numbers. This gives the familiar sequence of nuclear states: $1 \mathrm{~s}_{1 / 2}, 1 \mathrm{p}_{3 / 2}$, $1 \mathrm{p}_{1 / 2}, 2 \mathrm{~s}_{1 / 2}, 1 \mathrm{~d}_{5 / 2}, 1 \mathrm{~d}_{3 / 2}$ and so on. When a state is completely filled with neutrons and protons a nucleus of exceptional stability is formed, and this naturally gives the shell structure and associated "magic" numbers of neutrons or protons.

The stable structures formed by magic

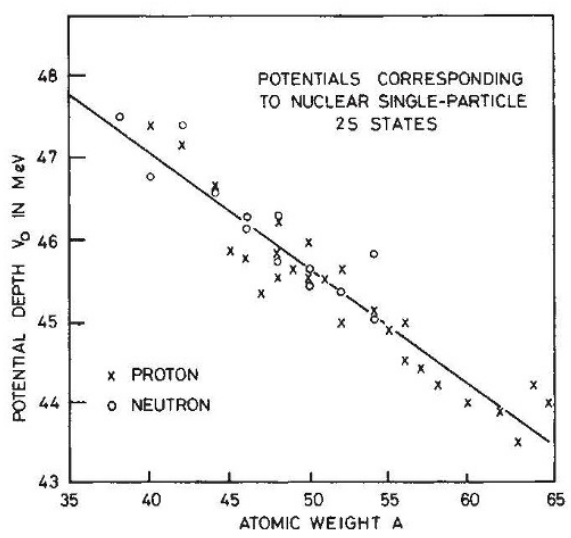

Potentials fitted to individual $2 \mathrm{~s}$ hole states compared with the average value. The dependence on the nuclear symmetry parameter has been removed using the best value of the corresponding term in the potential.

numbers of neutrons and protons are hardly disturbed when a single nucleon is added to them, so that the properties of such nuclei are in many respects those of a single nucleon coupled to a relatively inert core. The low-lying states are just those expected from the simple shell model, and the occupancy of the orbit as measured by a nucleon transfer reaction is close to unity. Nuclei with one particle less than a closed shell also show these single-particle states, as they are called.

If additional nucleons are added or taken away from such nuclei the nucleons outside the core can couple together in many ways, giving much more complicated sequences of nuclear levels, and the simplicity of the model is lost. The single-particle states are each split into a number of fragments, spread over several million electron volts of excitation energy. It has, however, recently been found by Millener and Hodgson (Nucl. Phys., A209, 59; 1973) that if the centroid of the fragmented state is calculated by weighting the energies of the fragments by their strengths as determined by one-nucleon transfer reactions, then these centroid energies behave in a systematic way from one nucleus to the next, even far away from the closed shells. This shows that the forces which fragment the states are unable to change their centroid energies significantly.

It is possible to obtain these energies as eigenvalues of a simple one-body potential similar to the real part of the optical potential used to describe the elastic scattering of nucleons by nuclei. The depth of the potential depends linearly on the nuclear symmetry parameter $(N-Z) / A$ and on $A$ itself. Each state can be followed from nucleus to nucleus across the periodic table until ultimately it is at too high or too low an energy to be measured accurately. The optimum parameters of the potential can thus be determined for each state individually and the state dependence is found to be quite small.

The optimum single-particle potentials give energies for the centroids of the single-particle states that agree with the experimental values to within about 0.2 to $0.3 \mathrm{MeV}$, which is comparable to the accuracy of the experimental measurements. The consistency between the potentials fitted to each state and the best fit potentials is shown for the 2 s hole states in the figure, and similar results are found for the other states.

These potentials can be used to calculate the wavefunctions of all the nucleons, and by summing their squared moduli $|\Psi|^{2}$ one can obtain the nuclear matter and charge distributions. The latter can be checked against the accurate values obtained from analyses of the elastic scattering of electrons by nuclei, and the agreement is within the experimental uncertainties.

\section{PLANT TAXONOMY}

\section{New World Bamboos}

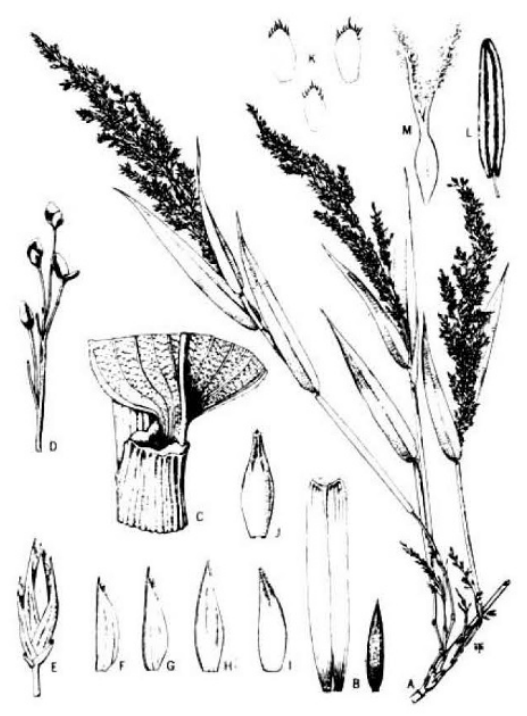

ON April 15, 1970, Floyd A. McClure died at work in his bamboo garden while removing a plant to give to a young friend. Before his death he had almost completed a revision of the genera of New World bamboos ; T. R. Soderstrom, a colleague of McClure's at the Smithsonian Institution, was fortunately able to complete the work and it has now appeared-with descriptions of seventeen genera, including four new genera and species-in the series Smithsonian Contributions to Botany (No. 9; 1973). This figure shows the form and parts of the type species, Swallenochloa subtessellata, of one of the new genera. 